\title{
DESEMPENHO DE DERRIÇADORA PORTÁTIL DE FRUTOS DO CAFEEIRO ${ }^{1}$
}

\section{CRISTIANO M. A. DE SOUZA ${ }^{2}$, DANIEL M. DE QUEIROZ ${ }^{3}$, FRANCISCO DE A. DE C. PINTO ${ }^{3}$, MAURI M. TEIXEIRA ${ }^{4}$}

\begin{abstract}
RESUMO: Este trabalho avaliou o desempenho de uma derriçadora portátil para cafeicultura de montanha durante a colheita de frutos de cafeeiros Catucaí e Catuaí, em vários estádios de desenvolvimento. Foram determinados as características dos cafeeiros, as curvas características do motor da derriçadora, a capacidade e a eficiência de derriça, o consumo de energia, a desfolha e o nível de ruído emitido pela máquina. A derriçadora portátil teve desempenho satisfatório por apresentar baixa desfolha e número de galhos quebrados, capacidade de derriça regular, baixo consumo específico de energia e elevada eficiência de derriça. O nível de ruído aumentou com o aumento da potência requerida na derriça.
\end{abstract}

PALAVRAS-CHAVE: energia elétrica, dano mecânico, ergonomia.

\section{PERFORMANCE OF FRUITS PORTABLE HARVESTER IN THE DETACHMENT OF COFFEE PLANTS}

\begin{abstract}
The portable harvester performance was evaluated for mountain coffee during the detachment of the fruits in different ages of the plants. The performance evaluation of the portable harvester was accomplished with four ages of plants and two coffee varieties. The coffee plant characteristics, the curve characteristics of the portable harvester engine, the detachment capacity and efficiency, the specific energy consumption, the mechanical damage and the noise level emitted by the machine were evaluated. The portable harvester held out a satisfactory performance by presenting less mechanical damage and number of broken branches, a regular detachment capacity, low specific energy consumption and high detachment efficiency. The noise level increased with the increasing of the power requirement.
\end{abstract}

KEYWORDS: electric power, mechanical damage, ergonomic.

\section{INTRODUÇÃO}

Dentre as etapas da produção do café, a colheita tem se constituído na operação mais onerosa da cultura, por exigir maiores contingentes de mão-de-obra, representando cerca de $50 \%$ do total empregado anualmente, podendo atingir até 35\% dos custos diretos da produção (MATIELLO et al., 2002).

A derriça do fruto do cafeeiro pode ser seletiva e total, podendo essas operações serem realizadas de forma manual, semimecanizada e mecanizada (SILVA et al., 1997). Na derriça seletiva, são colhidos apenas os frutos maduros, enquanto, na total, cada árvore é colhida de uma única vez e os frutos apanhados em todos os estádios de maturação. A derriça total é a prática mais usada na colheita de frutos do cafeeiro no Brasil.

\footnotetext{
${ }^{1}$ Extraído da tese de doutorado do primeiro autor.

${ }^{2}$ Eng ${ }^{0}$ Agrícola, D.Sc., Pesquisador DCR, UFMS/DCA, Dourados - MS, csouza@ ceud.ufms.br

${ }^{3}$ Ph.D., Prof. Adjunto, DEA/UFV, Viçosa - MG, queiroz@ufv.br e facpinto@ufv.br

${ }^{4}$ D.Sc., Prof. Adjunto, DEA/UFV, Viçosa - MG, mauri@ufv.br

Recebido pelo Conselho Editorial em: 14-10-2004

Aprovado pelo Conselho Editorial em: 11-10-2005
} 
A opção brasileira pelo sistema de derriça total prende-se à necessidade de baixar os custos de produção, uma vez que a colheita seletiva pode inviabilizar a cafeicultura, em virtude de seus altos custos, pois nesse sistema a colheita pode ser realizada em várias etapas durante o período.

Cerca de $90 \%$ das áreas cafeeiras ainda são colhidas manualmente e, por esse motivo, nos últimos anos, têm sido introduzidas derriçadoras portáteis, que são máquinas apropriadas para pequenos e médios produtores de café e para regiões com declividade superior a 20\% (MATIELLO et al., 2002).

As derriçadoras portáteis têm sido acionadas por sistema pneumático, motor de combustão interna ou motor elétrico. As potências de acionamento desses sistemas estão acima de $735 \mathrm{~W}$ para sistemas pneumáticos (PORTO, 1996) e motores de combustão interna (BARBOSA et al., 2005) e de $500 \mathrm{~W}$ para motores elétricos (CARVALHO et al., 2000).

Ensaios realizados por CARVALHO et al. (2000) têm demonstrado que as derriçadoras portáteis existentes no mercado ainda podem ter seu desempenho melhorado, podendo-se obter maior capacidade e eficiência de derriça e menor desfolhamento do cafeeiro. Portanto, torna-se necessária a realização de estudos a fim de torná-las mais viáveis técnica e economicamente, principalmente para a realidade da cafeicultura de montanha.

A ação das máquinas de colheita sobre o cafeeiro ocasiona danos, como desfolha, quebra e descorticamento de galhos e tronco (ARISTIZÁBAL-TORRES et al., 2000). BARROS et al. (1995), estudando o efeito da colheita realizada por uma derriçadora sobre os danos provocados à planta do cafeeiro, observaram que a colheita mecânica proporcionou desfolha semelhante à manual, tendo provocado quatro vezes mais ramos quebrados. As máquinas que proporcionam baixo índice de desfolhamento devem ser preferidas, pois além de preservarem a estrutura arbórea da planta, também podem garantir um produto de melhor qualidade, com menor presença de impurezas.

Quando a máquina proporciona conforto ao operador, seu desempenho aumenta sensivelmente, portanto é de fundamental importância conhecer o nível de ruído produzido por máquinas quando se pretende melhorar as condições de trabalho do operador. CUNHA et al. (2002) avaliaram os níveis de ruído emitido por três derriçadoras portáteis, concluindo que apresentaram níveis de ruído acima dos limites estabelecidos pela Norma NB 95 (NBR 10152) e pela NR-15 (CLT).

Considerando a limitada informação na bibliografia científica sobre as derriçadoras portáteis acionadas por motor elétrico e devido ao seu potencial de utilização na cafeicultura de montanha, este trabalho teve por objetivo avaliar o desempenho de uma derriçadora portátil durante a colheita de frutos do cafeeiro.

\section{MATERIAL E MÉTODOS}

O trabalho foi realizado no Departamento de Engenharia Agrícola da Universidade Federal de Viçosa e na Fazenda "Nô da Silva", localizada no município de Cajuri - MG.

Os ensaios de campo permitiram analisar o desempenho de uma derriçadora portátil de contato, acionada por motor elétrico, formada por um conjunto de 11 hastes curvilíneas oscilantes, sistema de engrenagens para a transmissão de potência e sistema de alimentação por bateria. A derriçadora apresentava $110 \mathrm{~mm}$ de comprimento das hastes oscilantes, $5 \mathrm{~mm}$ de diâmetro das hastes metálicas e 6 $\mathrm{mm}$ das emborrachadas, $1,2 \mathrm{~kg}$ de massa, $5,0 \mathrm{~kg}$ de massa da bateria e relação de transmissão de 12:50. A tensão da bateria era de $12 \mathrm{~V}$, e o consumo médio de $5 \mathrm{Ah}$. Um circuito de proteção disposto entre a bateria e o motor elétrico limitava a corrente elétrica que passava pelo motor em $15 \mathrm{~A}$. Embora a bateria fosse costal, ela possuía um cabo espiralado de $5,50 \mathrm{~m}$, possibilitando que fosse deixada sobre o terreno, facilitando o trabalho do operador. 
Para caracterizar em laboratório o comportamento do motor elétrico da derriçadora em função de sua rotação, foram determinadas a tensão e a corrente elétrica para vários carregamentos do motor, utilizando-se de um freio Prony. A rotação do motor foi determinada utilizando um sensor de pulso conectado a um sistema automático de aquisição de dados, por meio do qual também foram determinadas a tensão e a corrente elétrica. O sistema de aquisição automática de dados utilizado foi um HBM Spider8 e o programa computacional Catman.

Na determinação da potência elétrica produzida pelo motor, utilizou-se da seguinte equação:

$\mathrm{w}=\mathrm{AV}$

em que,

W - potência elétrica no motor, W;

A - corrente elétrica do sistema, A, e

V - tensão da bateria, V.

O torque no motor foi determinado em função da potência elétrica e de sua rotação, não sendo considerada sua eficiência, conforme a seguinte equação:

$$
\mathrm{T}=\frac{60 \mathrm{w}}{2 \pi \mathrm{z}}
$$

em que,

$\mathrm{T}$ - torque produzido no motor, $\mathrm{Nm}$, e

$\mathrm{z}$ - velocidade angular do motor, rpm.

Para caracterizar a planta do cafeeiro, foram medidos o diâmetro do tronco, o comprimento, o diâmetro e número dos galhos e a altura do cafeeiro, bem como determinada sua carga pendente. $\mathrm{O}$ diâmetro dos troncos foi medido no terço inferior, médio e superior. Os cafeeiros caracterizados foram selecionados aleatoriamente, num total de 160. Utilizaram-se trena e paquímetro marca Mitutoyo, com resolução de $0,05 \mathrm{~mm}$ nas medições. A carga pendente das plantas foi determinada somando a massa de frutos derriçados e os não-derriçados.

Durante os testes de campo, foram medidos a corrente e a tensão elétrica do motor para a determinação da potência elétrica da derriçadora, o tempo de derriça dos frutos do cafeeiro, a massa de frutos colhidos, a massa de frutos não-derriçados, a massa de folhas desprendidas e o número de galhos quebrados. Foram determinados a capacidade de derriça, a eficiência de derriça, o consumo de energia e o nível de ruído.

A capacidade de derriça foi determinada conforme a seguinte relação:

$$
\mathrm{C}_{\mathrm{d}}=60 \frac{\mathrm{m}_{\mathrm{d}}}{\mathrm{t}_{\mathrm{d}}}
$$

em que,

$\mathrm{C}_{\mathrm{d}}$ - capacidade de derriça, $\mathrm{kg} \mathrm{h}^{-1}$;

$\mathrm{m}_{\mathrm{d}}$ - massa de frutos derriçados, $\mathrm{kg}$, e

$t_{d}$ - tempo de derriça, min.

A eficiência de derriça foi obtida conforme a seguinte expressão:

$$
\xi_{\mathrm{d}}=100 \frac{\mathrm{m}_{\mathrm{d}}}{\mathrm{m}_{\mathrm{d}}+\mathrm{m}_{\mathrm{p}}}
$$

em que,

$\xi_{d}$ - eficiência de derriça, \%, e

$\mathrm{m}_{\mathrm{p}}$ - massa dos frutos não-derriçados, $\mathrm{kg}$. 
Os danos provocados pela derriça mecânica foram quantificados conforme se segue:

$$
D_{f}=\frac{m_{f}}{m_{d}}
$$

em que,

$\mathrm{D}_{\mathrm{f}}$ - desfolha provoca ao cafeeiro, $\mathrm{g} \mathrm{kg}^{-1}$, e

$\mathrm{m}_{\mathrm{f}}$ - massa de folhas desprendidas e ramos quebrados, $\mathrm{g}$.

O consumo específico de energia da derriçadora foi determinado conforme eq.(6):

$$
\mathrm{C}_{\mathrm{e}}=\frac{\mathrm{w}_{\mathrm{d}}}{\mathrm{C}_{\mathrm{d}}}
$$

em que,

$\mathrm{C}_{\mathrm{e}}$ - consumo específico de energia, $10^{-3} \mathrm{kWh} \mathrm{kg}^{-1}$, e

$\mathrm{w}_{\mathrm{d}}$ - potência elétrica da derriçadora, $\mathrm{kW}$.

Foram determinados os níveis de ruído próximo ao ouvido do operador com medidor de pressão sonora (decibelímetro) da marca MINIPA, modelo MSL-1350, no circuito de resposta lenta e de equalização "A". As análises dos dados foram realizadas, comparando-os com os limites de conforto estabelecidos pela Norma NBR 10152 (ABNT, 1987), que estabelece os níveis máximos de ruído que proporcionam o mínimo de conforto aos ocupantes de um ambiente e com os limites estabelecidos pela portaria $\mathrm{n}^{\mathrm{o}}$ 3.214, de 8 de junho de 1978, publicada como Norma Regulamentadora NR-15 da Consolidação das Leis do Trabalho.

Para analisar os dados de desempenho da derriçadora, foi montado um experimento em esquema fatorial, com quatro idades e duas variedades do cafeeiro, segundo o delineamento inteiramente casualizado, com quatro repetições. Cada repetição foi composta da derriça contínua de cinco plantas consecutivas, selecionadas ao acaso na lavoura.

Os dados obtidos da avaliação da derriçadora foram submetidos à análise de variância e de regressão, sendo os modelos selecionados com base no coeficiente de determinação e na significância dos coeficientes de regressão, utilizando-se do teste t, a 0,05 de probabilidade. Foi realizado o teste de Tukey, a 0,05 de probabilidade, na comparação entre médias de variedades. As análises estatísticas foram realizadas, utilizando-se do programa SAEG (RIBEIRO JÚNIOR, 2001), versão 8.

\section{RESULTADOS E DISCUSSÃO}

A potência elétrica do motor diminuiu quando foi aumentada sua rotação [eq.(7) e Figura 1a]. O maior valor de potência estimada durante o ensaio do motor elétrico foi de $173 \mathrm{~W}$, para a rotação de $4.000 \mathrm{rpm}$, enquanto o menor foi de $15,7 \mathrm{~W}$ para rotação de $15.000 \mathrm{rpm}$.

Não foram obtidos menores valores de rotação do motor pelo fato de o ensaio ter sido realizado com o circuito de proteção conectado ao sistema de alimentação do motor da máquina. Verificou-se que sua utilização limitou a potência do motor no valor obtido, na rotação de aproximadamente $4.000 \mathrm{rpm}$.

O torque do motor apresentou comportamento exponencial com o aumento da rotação [eq.(8) e Figura 1b], tendo sido o maior torque de 0,4 $\mathrm{Nm}$.

$$
\begin{array}{ll}
\hat{\mathrm{W}}=230,21-0,0143 \mathrm{z} & \mathrm{R}^{2}=0,99 \\
\hat{\mathrm{T}}=1,3232 \mathrm{e}^{-0,0003 \mathrm{z}} & \mathrm{R}^{2}=0,98
\end{array}
$$

em que, 
$\hat{\mathrm{W}}$ - potência elétrica estimada, $\mathrm{W}$, e

$\hat{\mathrm{T}}$ - torque no eixo do motor elétrico estimado, $\mathrm{Nm}$.

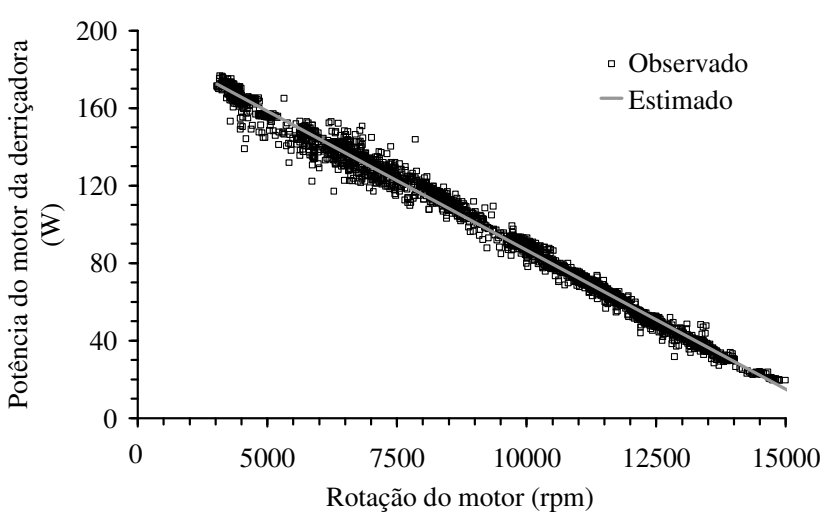

(a)

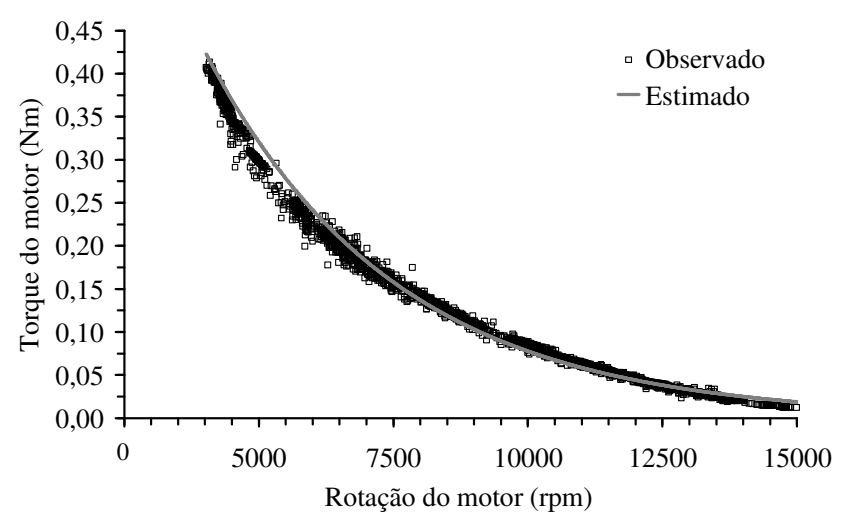

(b)

FIGURA 1. Potência (a) e torque (b) do motor elétrico em função da rotação.

As plantas que se encontravam em estádio de desenvolvimento mais avançado, apresentaram maiores valores para altura, diâmetro dos ramos ortotrópicos, comprimento e número de ramos plagiotrópicos, não tendo havido influência sobre o diâmetro médio dos ramos plagiotrópicos. Não houve diferença entre as variedades nas idades de desenvolvimento de três e dez anos. Não houve diferença entre as cargas pendentes das duas variedades de cafeeiros com idades de quatro e dez anos, enquanto, nas idades de três e seis anos, o cafeeiro Catucaí apresentou maior e menor valor que o cafeeiro Catuaí, respectivamente (Tabela 1).

TABELA 1. Características das plantas em função da idade e da variedade dos cafeeiros.

\begin{tabular}{|c|c|c|c|c|}
\hline \multirow{2}{*}{ Variedade do Cafeeiro } & \multicolumn{4}{|c|}{ Idade do Cafeeiro (anos) } \\
\hline & 3 & 4 & 6 & 10 \\
\hline & \multicolumn{4}{|c|}{ Altura (m) } \\
\hline Catucaí & $1,60 \mathrm{Ab}$ & $1,85 \mathrm{Ab}$ & $2,28 \mathrm{Aa}$ & $2,23 \mathrm{Aa}$ \\
\hline \multirow[t]{2}{*}{ Catuaí } & $1,59 \mathrm{Ac}$ & $1,91 \mathrm{Ab}$ & $1,97 \mathrm{Bab}$ & $2,19 \mathrm{Aa}$ \\
\hline & \multicolumn{4}{|c|}{ Diâmetro $(\mathrm{mm})$ do ramo ortotrópico } \\
\hline Catucaí & $22,82 \mathrm{Ab}$ & $24,08 \mathrm{Ac}$ & $30,68 \mathrm{Ab}$ & $37,37 \mathrm{Aa}$ \\
\hline \multirow[t]{2}{*}{ Catuaí } & $23,34 \mathrm{Ab}$ & $25,69 \mathrm{Ab}$ & $25,69 \mathrm{Bb}$ & $34,68 \mathrm{Aa}$ \\
\hline & \multicolumn{4}{|c|}{ Diâmetro $(\mathrm{mm})$ do ramo plagiotrópico } \\
\hline Catucaí & $6,07 \mathrm{Aa}$ & $8,84 \mathrm{Aa}$ & $5,04 \mathrm{Aa}$ & $6,47 \mathrm{Aa}$ \\
\hline \multirow[t]{2}{*}{ Catuaí } & $5,92 \mathrm{Aa}$ & $4,78 \mathrm{Ba}$ & 7,19 Aa & $6,01 \mathrm{Aa}$ \\
\hline & \multicolumn{4}{|c|}{ Comprimento $(\mathrm{cm})$ do ramo plagiotrópico } \\
\hline Catucaí & $54,18 \mathrm{Ac}$ & $57,33 \mathrm{Abc}$ & $69,80 \mathrm{Aab}$ & $78,15 \mathrm{Aa}$ \\
\hline \multirow[t]{2}{*}{ Catuaí } & $65,40 \mathrm{Aab}$ & $58,63 \mathrm{Ab}$ & $61,63 \mathrm{Aab}$ & $74,43 \mathrm{Aa}$ \\
\hline & \multicolumn{4}{|c|}{ Número de ramos plagiotrópico } \\
\hline Catucaí & $71 \mathrm{Ac}$ & $86 \mathrm{Abc}$ & $119 \mathrm{Aa}$ & $104 \mathrm{Aab}$ \\
\hline \multirow[t]{2}{*}{ Catuaí } & $73 \mathrm{Ab}$ & $89 \mathrm{Ab}$ & $95 \mathrm{Bb}$ & $120 \mathrm{Aa}$ \\
\hline & \multicolumn{4}{|c|}{ Carga pendente $\left(\mathrm{kg}\right.$ planta $\left.^{-1}\right)$} \\
\hline Catucaí & 3,79 Aab & $3,39 \mathrm{Aa}$ & $4,28 \mathrm{Aab}$ & $5,44 \mathrm{Ab}$ \\
\hline Catuaí & $2,28 \mathrm{Bc}$ & $4,02 \mathrm{Abc}$ & $6,79 \mathrm{Ba}$ & $5,28 \mathrm{Aab}$ \\
\hline
\end{tabular}

Médias seguidas por letras iguais, maiúsculas na coluna e minúsculas na linha, não diferem entre si, pelo teste de Tukey, a 0,05 de probabilidade. 
A capacidade de derriça apresentou comportamento distinto entre as duas variedades, na idade de três anos, enquanto não houve diferença para maiores idades. Os maiores valores de capacidade de derriça foram observados nas menores idades do cafeeiro, três anos para a variedade Catucaí e quatro anos na Catuaí (Tabela 2).

TABELA 2. Capacidade de derriça $\left(\mathrm{kg} \mathrm{h}^{-1}\right)$ em função da idade do cafeeiro, para as duas variedades

\begin{tabular}{ccccc}
\hline \multirow{2}{*}{ Variedade do Cafeeiro } & \multicolumn{4}{c}{ Idade do Cafeeiro (anos) } \\
\cline { 2 - 5 } & 3 & 4 & 6 & 10 \\
\hline Catucaí & $51,30 \mathrm{Aa}$ & $43,82 \mathrm{Aa}$ & $28,05 \mathrm{Ab}$ & $31,44 \mathrm{Ab}$ \\
Catuaí & $25,62 \mathrm{Bb}$ & $38,16 \mathrm{Aa}$ & $34,58 \mathrm{Aab}$ & $31,04 \mathrm{Aab}$ \\
\hline
\end{tabular}

Médias seguidas por letras iguais, maiúsculas na coluna e minúsculas na linha, não diferem entre si, pelo teste de Tukey, a 0,05 de probabilidade.

A capacidade de derriça não foi influenciada pela carga pendente do cafeeiro, indicando que pode haver outros parâmetros influenciando-a, tais como, o tamanho do cafeeiro, o número total de galhos e de ramificações, estande de plantas, tensão elétrica de acionamento do motor, etc.

Não houve diferença significativa entre as variedades do cafeeiro sobre a eficiência da derriça, independentemente de sua idade. Cafeeiros maiores de quatro anos apresentaram eficiências semelhantes (Tabela 3). O aumento da eficiência de derriça com a idade do cafeeiro pode ser devido à melhor manipulação da máquina, favorecida pelo afastamento dos frutos do tronco conforme se desenvolve a planta. Houve aumento da eficiência de derriça com o aumento da carga pendente de frutos por planta (Figura 2).

TABELA 3. Eficiência de derriça (\%) em função da idade do cafeeiro, para as duas variedades.

\begin{tabular}{ccccc}
\hline \multirow{2}{*}{ Variedade do cafeeiro } & \multicolumn{4}{c}{ Idade do cafeeiro (anos) } \\
\cline { 2 - 5 } & 3 & 4 & 6 & 10 \\
\hline Catucaí & $97,9 \mathrm{Aa}$ & $99,2 \mathrm{Ab}$ & $98,8 \mathrm{Ab}$ & $99,2 \mathrm{Ab}$ \\
Catuaí & $98,0 \mathrm{Aa}$ & $99,4 \mathrm{Ab}$ & $98,8 \mathrm{Ab}$ & $99,3 \mathrm{Ab}$ \\
\hline
\end{tabular}

Médias seguidas por letras iguais, maiúsculas na coluna e minúsculas na linha, não diferem entre si, pelo teste de Tukey, a 0,05 de probabilidade.

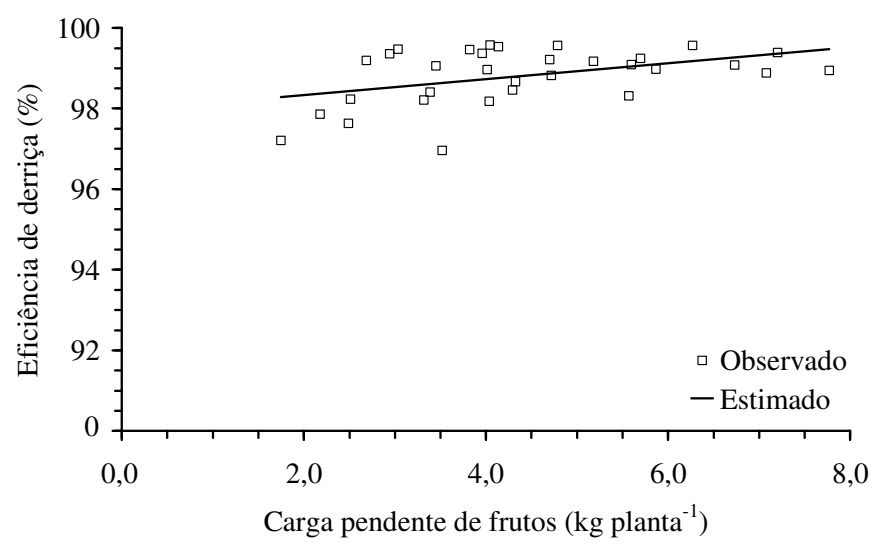

FIGURA 2. Eficiência de derriça em função da carga pendente de frutos do cafeeiro.

Observou-se, na massa de frutos não-derriçados, que a maioria estava no estádio de maturação verde, seguido pelos cereja e passas/secos, equivalendo a 46,7; 38,5 e 14,8\%, respectivamente. 
A desfolha ocasionada às duas variedades aumentou com o aumento de sua idade até oito anos, havendo, a partir daí, tendência de ligeira queda [Figura 3a e eq.(9)]. Não houve diferença significativa entre as variedades de cafeeiro. $\mathrm{O}$ aumento da carga pendente proporcionou aumento na desfolha (Figura 3b). A maior carga pendente de frutos preenchia mais os espaços entre as hastes da derriçadora e os galhos, causando maior possibilidade de queda de folhas por estrangulamento e/ou tração, além de fadiga pela vibração.

$$
\hat{D}_{\mathrm{f}}=-16,3716+43,519 \mathrm{I}_{\mathrm{d}}-2,8768 \mathrm{I}_{\mathrm{d}}^{2} \quad \mathrm{R}^{2}=0,62
$$

em que,

$\hat{\mathrm{D}}_{\mathrm{f}}$ - desfolha estimada do cafeeiro, $\mathrm{g}$ de folha por $\mathrm{kg}$ de frutos derriçados, e

$\mathrm{I}_{\mathrm{d}}$ - idade do cafeeiro, anos.

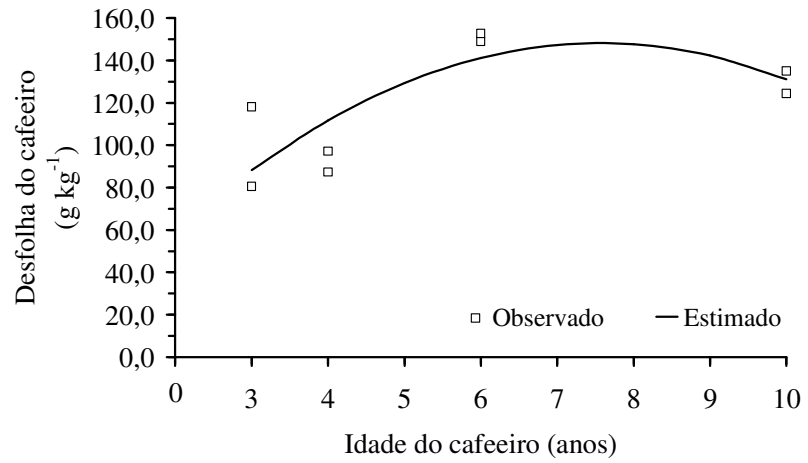

(a)

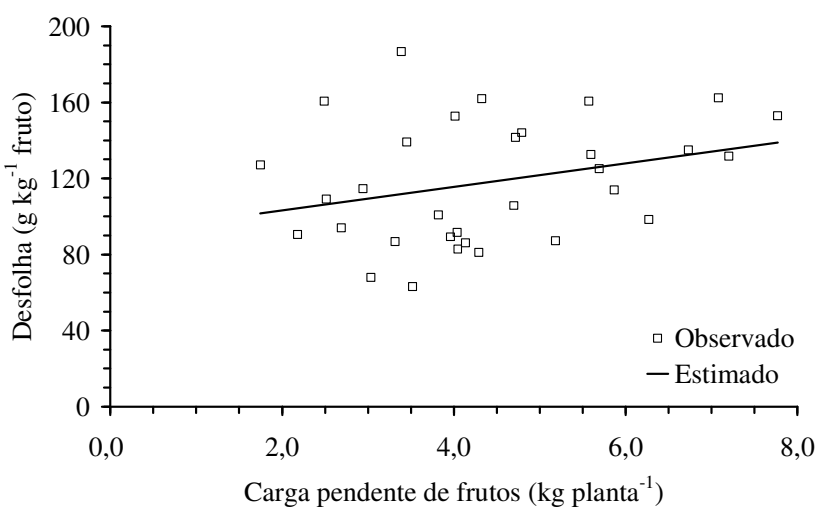

(b)

FIGURA 3. Desfolha do cafeeiro em função da idade (a) e da carga pendente (b).

O comportamento do número de galhos quebrados aumentou com a idade do cafeeiro [eq.(10) e Figura 4]. Esse comportamento era esperado, pois os cafeeiros mais velhos apresentam maior número de galhos que os mais jovens, aumentando a possibilidade de quebra dos ramos. Além disso, para alcançar os ramos mais altos, o operador tem de puxá-los e curvá-los, aumentando a quebra.

$$
\begin{array}{ll}
\hat{\mathrm{n}}_{\mathrm{f}}=1,5294+0,0729 \mathrm{I}_{\mathrm{d}} & \mathrm{R}^{2}=0,69
\end{array}
$$

em que,

$\hat{\mathrm{n}}_{\mathrm{f}}$ - número de galhos quebrados por massa de frutos derriçados.

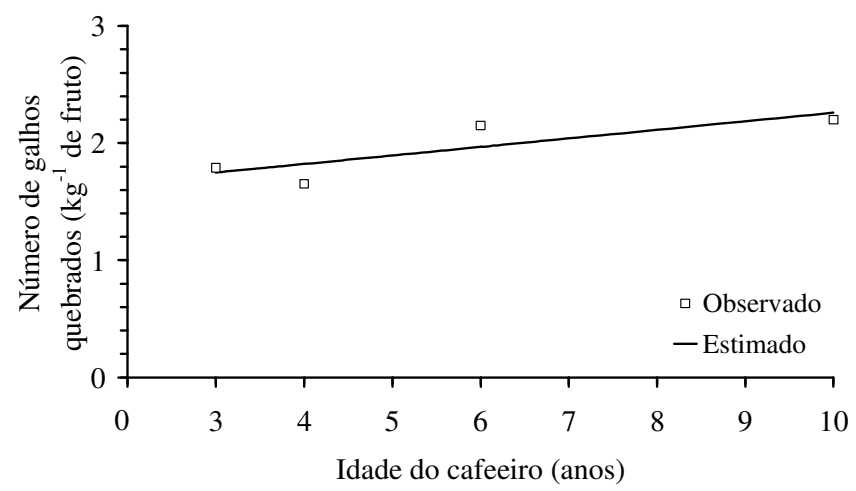

FIGURA 4. Número de galhos quebrados por massa de frutos derriçados em função da idade do cafeeiro. 
A potência requerida na derriça dos frutos do cafeeiro apresentou tendência quadrática com o incremento da idade da planta [Figura 5 e eq.(11)]. O maior valor da potência estimada requerida pela derriçadora foi de $86,3 \mathrm{~W}$, observado na idade de seis anos.

$$
\hat{\mathrm{W}}_{\mathrm{d}}=38,994+13,837 \mathrm{I}_{\mathrm{d}}-1,0163 \mathrm{I}_{\mathrm{d}}^{2}
$$

$$
\mathrm{R}^{2}=0,84(11)
$$

em que,

$\hat{\mathrm{W}}_{\mathrm{d}}$ - potência elétrica estimada requerida na derriça, $\mathrm{W}$.

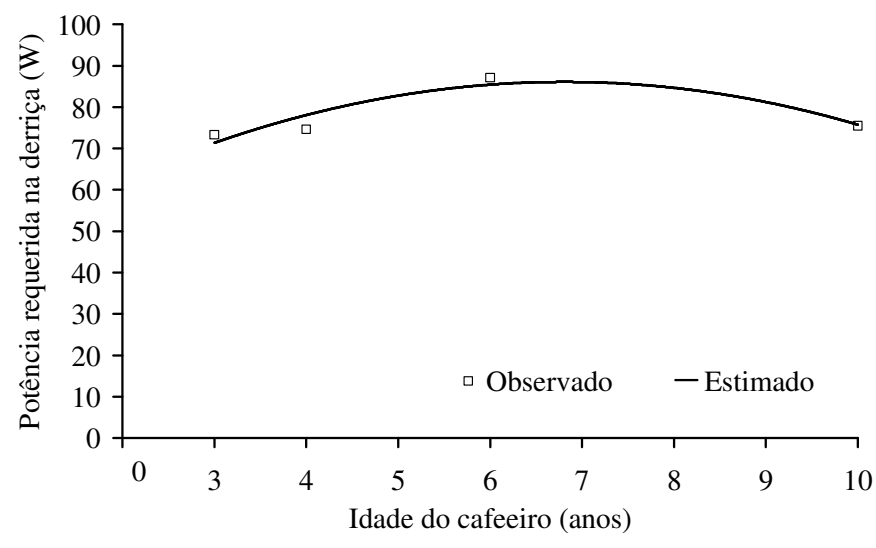

FIGURA 5. Potência elétrica da derriçadora em função da idade de cafeeiro.

O intervalo de confiança da potência exigida pela derriçadora, funcionando na rotação nominal do motor, foi de 36,1 $\pm 2,04 \mathrm{~W}$. No ensaio do motor (Figura 2), determinaram-se 15,4 W de potência na rotação nominal; sendo assim, pode-se dizer que a potência consumida pelas hastes e caixa de engrenagens é de aproximadamente 20,7 W.

De maneira geral, os valores requeridos de potência na derriça foram inferiores àqueles de derriçadoras que utilizam acionamento por motor à combustão interna (BARBOSA et al., 2005) e sistema pneumático (PORTO, 1996), incluindo o elétrico proposto por CARVALHO et al. (2000), mostrando grande potencial de uso na cafeicultura de montanha, onde se requer máquinas com menor consumo de energia.

O consumo específico de energia aumentou com a idade do cafeeiro, enquanto somente foi observada diferença significativa entre variedades, nas idades de três e seis anos (Tabela 4). Essa diferença de consumo específico de energia entre as variedades pode estar relacionada com a força de desprendimentos dos frutos, pois SAMPAIO (2000) observou que as forças requeridas no desprendimento dos frutos variaram com as variedades pesquisadas.

TABELA 4. Consumo específico de energia $\left(10^{-3} \mathrm{kWh} \mathrm{kg}^{-1}\right)$ da derriçadora portátil em função da idade e da variedade do cafeeiro.

\begin{tabular}{lcccc}
\hline \multirow{2}{*}{ Variedade do Cafeeiro } & \multicolumn{4}{c}{ Idade do Cafeeiro (anos) } \\
\cline { 2 - 5 } & 3 & 4 & 6 & 10 \\
\hline Catucaí & $1,46 \mathrm{Ac}$ & $1,78 \mathrm{Abc}$ & $3,17 \mathrm{Aab}$ & $2,46 \mathrm{Aabc}$ \\
Catuaí & $2,90 \mathrm{Bab}$ & $1,97 \mathrm{Abc}$ & $2,54 \mathrm{Bab}$ & $2,45 \mathrm{Aab}$ \\
\hline
\end{tabular}

Médias seguidas por letras iguais, minúsculas na linha e maiúsculas na coluna, não diferem entre si, pelo teste de Tukey, a 0,05 de probabilidade.

O incremento na potência requerida na derriça ocasionou aumento no nível de ruído emitido pela derriçadora em operação [eq.(12) e Figura 6]. Ambos os parâmetros estão altamente correlacionados com as características e propriedades do cafeeiro. 


$$
\hat{\mathrm{R}}_{\mathrm{d}}=-1536,2+40,126 \mathrm{w}_{\mathrm{d}}-0,2449 \mathrm{w}_{\mathrm{d}}^{2} \quad \mathrm{R}^{2}=0,96
$$

em que,

$\hat{\mathrm{R}}_{\mathrm{d}}$ - nível de ruído emitido pela derriçadora estimado, dBA.

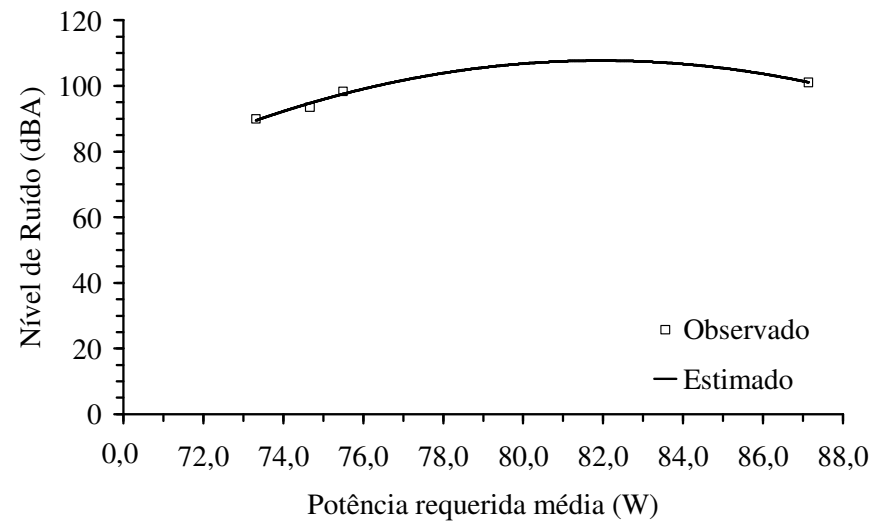

FIGURA 6. Nível de ruído emitido pela derriçadora em função da potência média requerida na derriça.

Os valores de níveis de ruído obtidos estão acima dos limites de conforto preestabelecidos pela norma NBR 10152 (ABNT, 1987) e a norma NR-15, que é de 85 dBA para 8 h de exposição diária, independentemente da idade e variedade do cafeeiro. De maneira geral, o nível de ruído emitido pela derriçadora avaliada, que variou entre 95,7 e 102,5 dBA, foi menor que aqueles obtidos por CUNHA et al. (2002).

\section{CONCLUSÕES}

O aumento na rotação do motor elétrico proporcionou diminuição na potência e comportamento exponencial no torque.

A capacidade de derriça da máquina apresentou comportamento distinto entre as duas variedades com o aumento da idade do cafeeiro, sendo que a variedade Catucaí apresentou maior valor.

A eficiência de derriça não foi influenciada pela variedade do cafeeiro, enquanto o aumento na idade proporcionou ligeira melhoria.

A desfolha, os números de galhos quebrados, a potência requerida na derriça e o consumo específico de energia aumentaram com a idade do cafeeiro, independentemente da variedade.

O aumento da carga pendente do cafeeiro proporcionou aumento da eficiência da derriça e da desfolha, não tendo influenciado na capacidade de derriça.

O nível de ruído próximo ao ouvido do operador da máquina apresentou valores acima dos preestabelecidos pelas normas brasileiras.

\section{AGRADECIMENTOS}

Ao Conselho Nacional de Desenvolvimento Científico e Tecnológico (CNPq), pelas bolsas de estudo e de pesquisa concedidas. Ao Programa Nacional de Pesquisa e Desenvolvimento do Café (PNP\&D Café) e à Fundação de Amparo à Pesquisa do Estado de Minas Gerais (FAPEMIG), pelo suporte financeiro. 


\section{REFERENCIAS}

ARISTIZÁBAL-TORRES, I.D.; OLIVEROS-TASCÓN, C.E.; SANZ-URIBE, J.R.; MONTOYARESTREPO, E.C.; ALVAREZ-MEJÍA, F. Cosecha mecánica de café aplicando vibraciones circulares al tallo del cafeto. Cenicafé, Chinchiná, v.51, n.1, p.41-53, 2000.

ASSOCIAÇÃO BRASILEIRA DE NORMAS TÉCNICAS. NBR 10152 (NB 95): Níveis de ruído para conforto acústico. Rio de Janeiro, 1987. 4 p.

BARBOSA, J.A.; SALVADOR, N.; SILVA, F.M. Desempenho operacional de derriçadores mecânicos portáteis, em diferentes condições de lavouras cafeeiras. Revista Brasileira de Engenharia Agrícola e Ambiental, Campina Grande, v.9, n.1, p.129-32, 2005.

BARROS, U.V.; BARBOSA, C.M.; HERINGER, F.; MATIELLO, J.B. Teste com a derriçadora agromática, na colheita de café. In: CONGRESSO BRASILEIRO DE PESQUISAS CAFEEIRAS, 21., 1995, Curitiba. Resumos... Rio de Janeiro: IBC, 1995. p.124-5.

CARVALHO, L.T.; QUEIROZ, D.M.; TEIXEIRA, M.M.; FERNANDES, H.C. Desenvolvimento de uma derriçadora portátil para colheita de café. In: SIMPÓSIO DE PESQUISA DOS CAFÉS DO BRASIL, 1., 2000, Poços de Caldas. Resumos Expandidos... Brasília: Embrapa Café; Belo Horizonte: Minasplan, 2000. v.2, p.1109-13.

CUNHA, J.P.A.R.; FERNANDES, H.C.; BARBOSA, J.A. Avaliação dos níveis de ruído emitidos por derriçadoras portáteis para colheita de café. In: CONGRESSO BRASILEIRO DE ENGENHARIA AGRÍCOLA, 31., 2002, Salvador. Anais... Salvador: Associação Brasileira de Engenharia Agrícola, 2002. 1 CD-ROM.

MATIELLO, J.B.; SANTINATO, R.; GARCIA, A.W.R.; ALMEIDA, S.R.; FERNADES, D.R. Cultura de café no Brasil: novo manual de recomendações. Rio de Janeiro: MAPA/PROCAFÉ, 2002. $387 \mathrm{p}$.

PORTO, F.S.A. Desenvolvimento de uma colhedora portátil de café por sucção aerodinâmica. 1996. 97 f. Dissertação (Mestrado em Projetos e Materiais) - Universidade Estadual Paulista "Julio de Mesquita Filho", Guaratinguetá, 1996.

RIBEIRO JÚNIOR, J.I. Análises estatísticas no SAEG. Viçosa - MG: Editora UFV, 2001. 301 p. SAMPAIO, C.P. Determinação da força requerida para o desprendimento de frutos de café em diferentes estádios de maturação. 2000. 37 f. Dissertação (Mestrado em Armazenamento e Processamento de Produtos Vegetais) - Universidade Federal de Viçosa, Viçosa - MG, 2000.

SILVA, F.M.; CARVALHO, G.R.; SALVADOR, N. Mecanização da colheita do café. Informe Agropecuário, Belo Horizonte, v.18, n.187, p.43-54, 1997. 